\title{
Intervenções Nutricionais na Anemia Ferropriva
}

\section{Nutritional Strategies for Controlling Iron Deficiency Anemia}

\author{
Marly A. Cardoso ${ }^{1}$ \\ Marilene de V. C. Penteado'
}

\section{CARDOSO, M. A. E PENTEADO, M. V.C. Nutritional Strategies for Controlling Iron}

Deficiency Anemia. Cad. Saúde Públ., Rio de faneiro, 10 (2): 231-240, Apr/fun, 1994.

This review was elaborated in order to contribute to the planning of strategies for controlling iron

deficiency anemia in developing countries. The need for intervention should be determined by the degree of iron deficiency in the individual group and knowledge of its effects on quality of life, morbidity, and mortality. The most frequent approach is to provide iron supplementation during pregnancy, lactation, and early childhood as a basic primary healthcare measure.

Fortification and dietary modification are complementary approaches, and should be developed.

Key words: Iron Deficiency Anemia; Iron; Nutritional Status; Epidemiology

\section{INTRODUÇÃO}

A deficiência de ferro é o distúrbio nutricional mais prevalente no mundo inteiro e afeta mais de um bilhão de pessoas, principalmente mulheres em idade fértil e pré-escolares das áreas tropicais e subtropicais (Gillespie et al., 1991). Segundo estimativas da Organização Mundial de Saúde (OMS), a anemia afeta cerca de $30 \%$ da população mundial, e pelo menos metade desta prevalência global pode ser atribuída à deficiência de ferro (De Maeyer \& Adiéls-Tegman, 1985). Na América Latina, estima-se uma variação da prevalência de anemia de $13 \%$ em homens adultos a $30 \%$ ou mais em gestantes (De Maeyer et al., 1989). No Brasil, a deficiência de ferro tem sido reconhecida como importante causa de anemia há mais de duas décadas (Cook et al., 1971). As estimativas da prevalência de anemia por deficiência de ferro são escassas e geralmente sem informações sobre reservas orgânicas de ferro da população geral (Vannucchi et al., 1992). A maioria dos estudos disponíveis refere-se exclusivamente a crianças (Lira et al., 1985; Sigulem et al., 1978; Monteiro

${ }^{1}$ Departamento de Alimentos e Nutrição Experimental da Faculdade de Ciências Farmacêuticas da Universidade de São Paulo. Av. Prof. Lineu Prestes, 580, Caixa Postal 66355, São Paulo, SP, 05389-970, Brasil.
\& Szarfarc, 1987) e a gestantes (Szarfarc, 1974; Salzano et al., 1980), consideradas os grupos de maior vulne-rabilidade, ou a usuários de serviços de saúde (Salzano et al., 1985; Szarfarc, 1985), que não compõem necessariamente uma amostra representativa da população total. Em geral, verificam-se prevalências de anemia superiores a $50 \%$ na população de faixa etária entre seis meses e dois anos (Monteiro \& Szarfarc, 1987; Cardoso et al., 1992).

A necessidade de intervenções para o controle da prevalência da anemia por deficiência de ferro deve ser determinada pela magnitude da deficiência nutricional e pelo conhecimento de seus efeitos na qualidade de vida, na morbidade e na mortalidade. A deficiência de ferro é um estado no qual há redução da quantidade total de ferro e o fornecimento de ferro é insuficiente para atingir as necessidades de diferentes tecidos, incluindo as necessidades para formação de hemoglobina $(\mathrm{Hb})$ dos eritrócitos. A anemia por deficiência de ferro (ADF) é conceitualmente um dos resultados da deficiência de ferro e refere-se à condição de fornecimento insuficiente de ferro à medula óssea, com conseqüente redução da concentração sangüínea de $\mathrm{Hb}$ abaixo do limite inferior do intervalo de $95 \%$ de confiança da distribuição dos valores populacionais de $\mathrm{Hb}$, segundo sexo, idade e estado fisiológico (Hallberg \& Rossander-Hulthén, 1989). 


\section{CAUSAS E CONSEQÜÊNCIAS DA DEFICIÊNCIA DE FERRO}

A deficiência de ferro da dieta pode causar anemia principalmente na infância, adolescência e gravidez, períodos cujas necessidades nutricionais de ferro são altas. Porém, apesar de a dieta ser o principal fator, nem sempre é a única causa da anemia. Outras deficiências nutricionais, infestações parasitárias e infecções desempenham um papel importante em países tropicais (Kasili, 1990). Doenças hemolíticas congênitas, tais como anemia falciforme e talassemia, são encontradas em certas populações, particularmente na África, Ásia e algumas ilhas do Oceano Pacífico (De Mayer et al., 1989).

A conseqüência mais óbvia da deficiência de ferro é a anemia com todos os sintomas clínicos e seqüelas extensamente descritas na literatura (Klevay, 1992). A redução da concentração de $\mathrm{Hb}$ sangüínea compromete $\mathrm{o}$ transporte de oxigênio para os tecidos, reduzindo a capacidade de trabalho e o desempenho físico em indivíduos anêmicos (De Maeyer et al., 1989). As bases bioquímicas desse comprometimento não foram totalmente elucidadas, embora possam estar relacionadas a alterações do metabolismo aeróbico (Baynes \& Bothewell, 1990; Bates \& Powers, 1989).

A deficiência de ferro durante a gravidez tem sido relacionada ao aumento da morbidade e mortalidade de gestantes e do feto. A relação entre anemia da gestante e risco de nascimento de crianças prematuras parece específica da ADF (Scholl et al., 1992). A concentração de $\mathrm{Hb}$ em recém-nascidos correlaciona-se positivamente com níveis maternos de ferritina sérica (Hereberg et al., 1987; De Benaze et al., 1989).

Quando a deficiência de ferro ocorre durante os primeiros dois anos de vida, há evidências de atraso no desenvolvimento psicomotor e alterações de comportamente (Lozoff et al., 1987; Walter et al., 1989; Heywood et al.; 1989). Segundo alguns autores, estes efeitos parecem persistir após vários meses de tratamento com ferro (Lozoff et al., 1991; Walter, 1993). No entanto, há extensa discussão acerca da consistência e validade externa desses dados (Pollitt, 1991). Idjaradinata \& Pollitt (1993), em estudo randomizado e duplo cego, de- monstraram que crianças de 12 a 18 meses de idade com ADF alcançaram índices de desenvolvimento psicomotor equivalentes aos de crianças sem anemia, independente das reservas de ferro, após quatro meses de tratamento com sulfato ferroso. Outros estudos, entretanto, relacionam déficit psicomotor e pondoestatural com outras variáveis de morbidade (desnutrição protéicocalórica, diarréia, infecções), que devem ser controladas, além de se fazer a correção da deficiência de ferro (Aukett et al., 1986; Javaid et al., 1991).

\section{FORMAS E BENEFÍCIOS DA SUPLEMENTAÇÃO COM FERRO}

A deficiência de ferro e suas múltiplas conseqüências podem ser corrigidas através de medidas simples, de baixo custo e comprovada eficácia. A abordagem mais usual é fornecer ferro suplementar a gestantes, nutrizes e lactentes em programas de assistência primária à saúde. Apesar da eficácia comprovada desses programas, sua eficiência é às vezes muito baixa. Dificuldades relacionadas ao abastecimento, distribuição e consumo dos suplementos de ferro são identificadas na grande maioria dos programas da rede básica de saúde (Gillespie et al., 1991). O principal objetivo das intervenções nutricionais é aumentar as reservas orgânicas de ferro de uma população-alvo, dentro das limitações de recursos disponíveis (De Maeyer et al., 1989).

O controle de infecções maláricas e por helmintos, concomitantemente às intervenções nutricionais e ações básicas de saúde, representa uma importante estratégia na redução da prevalência da ADF. Em regiões endêmicas de malária, os benefícios do tratamento oral com ferro sobrepõem-se aos riscos relacionados à infecção (Gillespie et al., 1991).

\section{Suplementação Terapêutica}

$\mathrm{O}$ tratamento com ferro medicamentoso deve ser utilizado em todos os pacientes com diagnóstico clínico-laboratorial de anemia, uma vez que as modificações da dieta por si só não podem corrigir a $\mathrm{ADF}$. $\mathrm{O}$ tratamento de escolha é a administração oral de ferro. A ad- 
ministração parenteral deve ser reservada para pacientes com intolerância ao ferro oral (De Maeyer et al., 1989).

O sulfato ferroso é o sal de escolha devido ao seu baixo custo e alta biodisponibilidade. A dose de tratamento depende da severidade da anemia. Para gestantes e nutrizes, $60 \mathrm{mg}$ de ferro elementar três vezes por dia na anemia severa $(\mathrm{Hb}<7 \mathrm{~g} / \mathrm{dl})$; na anemia leve a moderada, mesma dose duas vezes por dia. Para lactentes, pré-escolares e escolares, recomenda-se a dose de $3 \mathrm{mg} / \mathrm{kg}$ e peso/dia de ferro elementar; para adolescentes e adultos, a dose é $60 \mathrm{mg}$ de ferro elementar duas vezes por dia para anemia moderada (Gillespie et al., 1991). A correção da anemia geralmente ocorre em seis semanas. Entretanto, a reposição das reservas de ferro ocorre em quatro a seis meses devido, principalmente, à diminuição da absorção de ferro após correção da anemia (Massey, 1992).

Os efeitos colaterais gastrintestinais, tais como náuseas, cólicas abdominais, tais como náuseas, cólicas abdominais, obstipação e/ou diarréia, são observados em 15 a $20 \%$ dos pacientes no tratamento oral com ferro. Estes efeitos parecem relacionados à dose, que, apesar de prolongar o tratamento, pode ser reduzida para atenuar os efeitos colaterais (Massey, 1992). Estudos laboratoriais recentes com seres humanos relatam redução dos efeitos colaterais e aumento da eficácia de uma nova forma de ferro oral - Hydrodynamically Balanced System (HBS) ou Gastric Delivery System (GDS). Esta cápsula é capaz de prolongar a liberação gástrica de sulfato ferroso durante várias horas sem efeitos colaterais. Resultados preliminares mostram que uma simples cápsula de HBS com $50 \mathrm{mg}$ de ferro eqüivale a três comprimidos de sulfato ferroso com $50 \mathrm{mg}$ de ferro elementar (Cook et al., 1990).

As preparações de ferro parenteral (ferrodextran - Imferon $\left({ }^{\circledR}\right)$ não devem ser utilizadas na assistência primária à saúde, mas são necessárias na terapêutica de indivíduos com anemia severa, vômitos ou resistência ao tratamento. Os riscos associados ao seu uso (complicações na malária) e o alto custo do produto e de sua distribuição restringem sua utilização somente sob supervisão médica (Raman et al., 1989; De Maeyer et al., 1989).
Os aspectos sobre toxicidade do ferro incluem a interferência na absorção de outros minerais (zinco, por exemplo), sobrecarga crônica de ferro devido à hemocromatose ou talassemia, carcinogênese e consumo acidental por crianças. A prevenção de sobrecarga de ferro na hemocromatose e nas talassemias inclui diagnóstico e tratamento adequados dos indivíduos portadores dessas doenças. Em relação à interferência na absorção de outros minerais, a absorção de zinco pode ser competitivamente inibida pelo sulfato ferroso em solução aquosa quando a concentração iônica total de ambos exceder $25 \mathrm{mg}$. Não há evidências dessa interação competitiva na presença de alimentos (Yip et al., 1985; Gillespie et al., 1991).

\section{Suplementação Preventiva}

A suplementação com ferro como medida preventiva tem grande chance de sucesso quando dirigida a grupos específicos. A cobertura de toda população é usualmente desnecessária, logisticamente impossível e proibitivamente cara. A suplementação preventiva deve ser dirigida a grupos vulneráveis como gestantes e lactantes, lactentes e pré-escolares. Para escolares, a suplementação é menos freqüentemente necessária, aconselhando-se a realização de rastreamento neste grupo etário (Gillespie et al., 1991).

\section{Gestantes}

A suplementação com ferro deve ser distribuída a todas as gestantes na assistência primária à saúde. Em áreas de baixa prevalência de anemia $(\mathrm{Hb}<11 \mathrm{~g} / \mathrm{dl}$ em menos de $20 \%$ das gestantes na segunda metade da gravidez), a dose preconizada pela OMS é de um comprimido de $60 \mathrm{mg}$ de ferro elementar por dia (200 $\mathrm{mg}$ de sulfato ferroso) com $250 \mathrm{mg}$ de ácido fólico; em áreas de alta prevalência, dois comprimidos por dia (120 mg de ferro elementar e 500 mg de ácido fólico) (De Maeyer et al., 1989).

A suplementação deve ocorrer principalmente durante a segunda metade da gravidez. Uma outra abordagem do controle da anemia na gravidez consiste no planejamento de intervenções dirigidas a adolescentes e mulheres antes da concepção, como também no intervalo intergestacional. Es- 
tudos-piloto neste grupo etário têm demonstrado o efeito benéfico da suplementação nas reservas de ferro antes da gravidez (Kaufer \& Casanueva, 1990; Ballin et al., 1992).

\section{Lactentes}

A prevenção da $\mathrm{ADF}$ em lactentes inclui cuidados de saúde e promoção do aleitamento materno nos primeiros seis meses de idade. A partir do sexto mês de idade, a dose para prevenção da ADF é de $1 \mathrm{mg}$ de ferro elementar/kg de peso/dia(Gillespie et al., 1991). No entanto, há recomendações recentes de suplementação com ferro a partir do quarto mês de idade para crianças em aleitamento materno exclusivo (Calvo et al., 1992; Oski, 1993). Crianças com baixo peso ao nascer $(<2,5 \mathrm{~kg})$ apresentam velocidade de crescimento pós-natal maior; por isso, recomenda-se $2 \mathrm{mg}$ de ferro elementar/kg de peso/dia a partir do segundo mês de idade(Olivares et al., 1992; Oski, 1993). Bebês com peso ao nascer inferior a $1 \mathrm{~kg}$ devem receber $4 \mathrm{mg} / \mathrm{kg}$ de peso/dia, e aqueles com peso entre 1 e $1,5 \mathrm{~kg}, 3 \mathrm{mg}$ de ferro elementar/kg de peso/dia durante o primeiro ano de vida(Oski, 1993).

\section{Pré-Escolares}

Quando a suplementação é necessária (em áreas de alta prevalência), a dose é $30 \mathrm{mg}$ de ferro elementar/dia. Para melhorar a adesão à suplementação, sugere-se esta dose por duas a três semanas, várias vezes ao ano (De Maeyer et al., 1989; Smith et al., 1989).

\section{Escolares}

Se a prevalência de ADF apresentar magnitude importante, o serviço de saúde em assistência primária e os professores poderão fornecer doses diárias de 30 a $60 \mathrm{mg}$ de ferro elementar/dia, dependendo da idade e do peso da criança (Hathirat et al., 1992).

\section{Avaliação dos Programas}

A ineficiência da suplementação com ferro deve-se em muitos casos a dois motivos principais: efeitos colaterais do ferro oral (que se relacionam à dose utilizada) e dificuldade em manter a motivação para seguimento do programa (Schultink et al., 1993). Um estudo na Índia no período de 1985 - 1986 mostrou que o índice médio de abandono do programa nacional de suplementação com ferro variou de 9 a $87 \%$. Destes, mais de $80 \%$ dos indivíduos referem a falta de suplementos como motivo para desistência e menos de $3 \%$ referem os efeitos colaterais (Gillespie et al., 1991). Em todos os grupos, a suplementação deve ser de curto prazo (não mais do que quatro a cinco meses). Enquanto isso, outras medidas terapêuticas e preventivas devem ser implementadas (fortificação de alimentos, modificações da dieta, intervenções básicas de saúde) (Trowbridge et al., 1993).

O perigo da suplementação de ferro em indivíduos com anormalidades genéticas foi muito enfatizado no passado. Os estados heterozigotos (como na anemia falciforme e b-talassemia minor) não são condições de sobrecarga de ferro, e as reservas orgânicas de ferro desses indivíduos refletem o estado geral da população. A alta freqüência desses genes não deve interferir no planejamento de medidas de suplementação e fortificação com ferro (Gillespie et al., 1991).

Há evidências epidemiológicas e bioquímicas do comprometimento do metabolismo de ferro na deficiência de vitamina $\mathrm{A}$. A administração de ferro e vitamina $\mathrm{A}$ em áreas de prevalência da deficiência desses dois nutrientes resulta em melhor resposta hematológica (Haschke \& Javaid, 1991; Trowbridge et al., 1993).

A avaliação do nível de adesão nos programas de suplementação é necessária para estimar sua eficiência. A avaliação não deve utilizar medidas diretas de consumo devido o seu alto custo (tempo, pessoal e equipamentos). Métodos alternativos incluem a contagem dos comprimidos devolvidos ou a informação pelo indivíduo ou responsável, que pode ser realizada diretamente através do agente de saúde ou pelo agendamento de retornos em função do número de comprimidos fornecidos (Gillespie et al., 1991).

\section{Modificações da Dieta}

Há dois tipos de ferro da dieta. Cerca de $90 \%$ do ferro dos alimentos estão na forma de sais de ferro, denominados ferro não-heme. O grau de 
absorção deste tipo de ferro é altamente variável e depende das reservas de ferro do indivíduo e de outros componentes da dieta. Os outros $10 \%$ do ferro da dieta estão na forma de ferro heme provenientes principalmente da hemoglobina e da mioglobina. $O$ ferro heme é bem absorvido e seu nível de absorção é pouco influenciado pelas reservas orgânicas de ferro ou por outros constituintes da dieta (Lerner \& Iancu, 1988).

Não se conhece exatamente o mecanismo de absorção e biodisponibilidade do ferro em dietas mistas cujos alimentos são consumidos em diferentes proporções e de maneira não-uniforme em cada refeição (Vannucchi et al., 1992). Os constituintes da dieta que interferem na biodisponibilidade do ferro não-heme do pool de ferro intraluminal podem ser classificados em estimuladores e inibidores da absorção de ferro.

Entre os fatores estimuladores da dieta estão as carnes e os ácidos orgânicos como o cítrico, málico, tartárico, lático e, principalmente, o ácido ascórbico. O efeito da carne como estimulador relaciona-se especificamente à proteína muscular. O mecanismo não foi completamente esclarecido mas pode estar relacionado à liberação de cisteína e de peptídios com cisteína durante o processo de digestão, formando quelatos peptídio-ferro de fácil absorção. $\mathrm{O}$ ácido ascórbico converte o ferro férrico em ferroso, tornando-o solúvel no meio alcalino do intestino delgado. Além disso, no $\mathrm{pH}$ ácido do estômago, o ácido ascórbico forma um quelato com o cloreto férrico, que permanece estável em pH alcalino (Bothwell et al., 1989). A suplementação com ácido ascórbico tem sido sugerida para melhorar a biodisponibilidade de ferro da dieta e aumentar as reservas orgânicas de ferro em mulheres em idade reprodutiva (Hunt et al., 1990).

Entre os inibidores da absorção estão os polifenóis, fitatos, fosfatos e oxalatos. Os polifenóis são metabólitos secundários de origem vegetal, ricos em grupos hidroxil fenólicos que formam complexos insolúveis com ferro. Polifenóis de alto peso melcular - os taninos presentes no chá e no café são os maiores inibidores da absorção de ferro dos alimentos (Bothwell et al., 1989). O cálcio, em pequenas quantidades, parece aumentar a absorção de ferro, mas grandes quantidades inibem a ab- sorção. Os fosfatos ligados ou não a proteínas formam complexos insolúveis com ferro e são os principais responsáveis pela baixa biodisponibilidade do ferro dos ovos, leite e derivados. Os filatos, presentes em muitos cereais, inibem a absorção do ferro não-heme da dieta através da formação de complexo insolúvel de fitato di e tetra-férrico (Lerner \& iancu, 1988; Bothwell et al., 1989).

O leite humano e o leite de vaca contém cerca de 0,5 a $1 \mathrm{mg}$ de ferro/l, mas com biodisponibilidades diferentes. A absorção do ferro do leite humano (cerca de 50\%) é exclusivamente alta, o que compensa sua baixa concentração. Por outro lado, somente cerca de $10 \%$ do ferro do leite de vaca são absorvidos. Acredita-se que a baixa biodisponibilidade do ferro do leite de vaca esteja relacionada à alta concentração de cálcio e fosfoproteínas, juntamente com a baixa concentração de vitamina $\mathrm{C}$, em relação à composição química do leite humano (Oski, 1993).

As dietas ocidentais contêm cerca de $6 \mathrm{mg}$ de $\mathrm{Fe} / 1000 \mathrm{kcal}$, estimando-se um consumo diário de 12 a $18 \mathrm{mg}$ de ferro em muitos indivíduos (Acosta et al., 1984; Bothwell et al., 1989). Entre a população brasileira, há evidências da associação entre prevalência de anemia e deficiência de ferro das dietas, uma vez que nestas a quantidade total e a biodisponibilidade de ferro parecem insuficientes para atender as necessidades de ferro de vários grupos etários (Vannucchi et al., 1992).

Em relação às modificações da dieta, Gillespie et al. (1991) sugerem três principais formas de se aumentar as reservas orgânicas de ferro através da dieta:

1) Aumentar o consumo de ferro heme.

2) Aumentar o consumo de vitamina $\mathrm{C}$ e outros estimuladores da absorção de ferro nas refeições.

3) Diminuir, durante as refeições, o consumo dos inibidores da absorção de ferro (chá, café, alguns cereais, leite e derivados).

\section{Fortificação de Alimentos}

A fortificação de alimentos não substitui necessariamente a suplementação com ferro nem as orientações sobre modificações da dieta, mas, 
se efetiva a longo prazo, pode aumentar as reservas de ferro de uma população. Os programas de fortificação necessitam da identificação de uma fonte de ferro biodisponível não-reativo e veículos (alimentos) adequados à fortificação. Em alguns casos, a fortificação pode ser dirigida a grupos vulneráveis, por exemplo, alimentos de desmame (Gillespie et al., 1991).

A fortificação com ferro é tecnicamente mais difícil, pois as formas biodisponíveis de ferro são quimicamente reativas e produzem muitas vezes efeitos indesejáveis quando adicionadas aos alimentos. Uma etapa crítica no delineamento de um programa de fortificação com ferro é a seleção de um composto que seja discreto e bem absorvido. Os compostos solúveis são mais bem absorvidos e quimicamente mais reativos também; os compostos com fosfato são pouco reativos porém apresentam baixa biodisponibilidade em seres humanos (Cook \& Reusser, 1983). O ferro carbonil, recentemente sugerido como efetivo na correção da ADF, é uma forma não-tóxica de ferro elementar com partículas muito pequenas obtidas de íons metálicos altamente purificados (Devasthali et al., 1991).

Em países em desenvolvimento, a abordagem da fortificação difere da adotada em países desenvolvidos devido à baixa biodisponibilidade de ferro da dieta e ao pequeno número de alimentos elegíveis processados de forma centralizada. A eficiência do programa dependerá do sistema de distribuição regional. Os pré-requisitos para um programa efetivo de fortificação incluem: compromisso a longo prazo, fonte de ferro biodisponível compatível e alimentos adequados de acordo com a legislação vigente. Um alimento veiculador adequado seria aquele com processamento centralizado, viável à fortificação em termos tecnológicos e econômicos, sem sofrer alterações de sabor, textura e aparência; que seja freqüentemente consumido pela população-alvo e disponível através de um sistema efetivo de distribuição (Gillespie et al., 1991).

Exemplos de veículos utilizados em programas internacionais incluem pão branco e integral, sal, açúcar, leite em pó integral, fórmulas infantis, biscoitos, curry, molhos à base de peixe e de soja, sucos de frutas aromatizados. Recomenda-se o uso de um composto de ferro juntamente com vitamina $\mathrm{C}$, apesar de seu alto custo e destruição no aquecimento (Sarma et al., 1992; Trowbridge et al., 1993).

No Chile, um programa nacional de distribuição de biscoitos fortificados com $6 \%$ de concentrado de $\mathrm{Hb}$ bovina (CHB) mostrou-se capaz de aumentar as reservas de ferro de crianças em idade escolar após três anos de instalação do programa. Foram distribuídos três biscoitos de $10 \mathrm{~g}$ por dia por criança, o equivalente a $1 \mathrm{mg}$ de ferro biodisponível (Walter et al., 1993b).

Lactentes são reconhecidamente susceptíveis ao desenvolvimento da $\mathrm{ADF}$, com período de maior vulnerabilidade entre 6 e 18 meses de idade. $\mathrm{O}$ uso de leite de vaca e a introdução tardia de alimentos sólidos podem ser identificados como condutas alimentares inconvenientes à prevenção da $\mathrm{ADF}$ na infância (Aggett et al., 1989; Silva et al., 1993; Forsyth et al., 1993). Devido à alta biodisponibilidade do ferro no leite humano, o aleitamento materno oferece maior proteção contra o desenvolvimento da ADF nos primeiros seis meses de vida; a partir desta idade, recomenda-se a suplementação com ferro ou o uso de alimentos infantis fortificados com ferro (Siimes \& Salmenperä, 1989; Pizarro et al., 1991; Walter et al., 1993a). Entretanto, no uso de fórmulas infantis é essencial a fortificação com ferro e vitamina C $(15 \mathrm{mg}$ e $100 \mathrm{mg}$, respectivamente, por $100 \mathrm{~g}$ de leite em pó) (Olivares et al., 1989; Dallman, 1990).

No Brasil, está tramitando no Senado um projeto de lei prevendo a inclusão de vitaminas e ferro em três alimentos básicos - açúcar, leite e farinha de trigo. Em 1990, uma reunião organizada pela OMS em Nova York definiu uma política internacional de combate às carências demicronutrientes (anemias carencias, hipovitaminose A e bócio endêmico). Com relação à $\mathrm{ADF}$, o governo brasileiro, entre outros países, assumiu o compromisso de reduzir em $1 / 3$ a prevalência de anemia em gestantes e mulheres em idade reprodutiva até o ano 2000 (Batista-Filho \& Diniz, 1993). Há estudos preliminares com alimentos enriquecidos em crianças pré-escolares (Nogueira et al., 1992). No entanto, estas experiências ainda não foram incrementadas em um programa nacional de fortificação de alimentos integrado com outras 
medidas de combate à $\mathrm{ADF}$ na assistência primária à saúde (suplementação terapêutica, preventiva e modificações da dieta).

\section{CONCLUSÕES}

O abastecimento inadequado dos suplementos de ferro e a baixa cobertura dos serviços de saúde são as maiores dificuldades encontradas em muitos programas de suplementação com ferro. A eficiência dos programas depende da cobertura e alcance do serviço de distribuição. $\mathrm{O}$ reconhecimento dos efeitos da deficiência de ferro na qualidade de vida deve envolver todos os níveis dos programas, inclusive os indivíduos afetados. A adesão ao regime de suplementação é uma dificuldade muito mais relacionada ao fornecimento dos suplementos e motivação para o tratamento do que aos efeitos colaterais indesejáveis. Recomenda-se maior cobertura de gestantes, lactantes e lactentes em áreas de risco e maior atenção à suplementação com ferro em crianças prematuras e de baixo peso ao nascer. Uma outra abordagem do controle da anemia ferropriva na gravidez consiste no planejamento de intervenções dirigidas a adolescentes e mulheres em idade reprodutiva. Em outros grupos etários, recomenda-se a suplementação seletiva através de rastreamento. Fortificação de alimentos e orientações sobre modificações da dieta representam medidas complementares e devem ser incrementadas. Em geral, acredita-se que a integração dessas intervenções pode apresentar melhor impacto.

\section{RESUMO}

CARDOSO, M. A. \& PENTEADO, M. V. C. Intervenções Nutricionais na Anemia Ferropriva. Cad. Saúde Públ., Rio de Janeiro, 10 (2): 231-240, abr/jun, 1994.

O objetivo deste estudo de revisão bibliográfica é fornecer subsídios para o planejamento e avaliação de medidas de combate à anemia ferropriva. A necessidade de intervenções para o controle da prevalência da anemia ferropriva deve ser determinada pela magnitude da defi- ciência nutricional e pelo conhecimento de seus efeitos na qualidade de vida, morbidade e mortalidade. A abordagem mais usual é fornecer ferro suplementar a gestantes, nutrizes e lactentes em programas de assistência primária à saúde, reconhecidamente os grupos de maior vulnerabilidade. A fortificação de alimentos e orientações sobre modificações da dieta representam medidas complementares e devem ser incrementadas.

Palavras-Chave: Anemia Ferropriva; Ferro; Estado Nutricional; Epidemiologia

\section{REFERÊNCIAS BIBLIOGRÁFICAS}

ACOSTA, A.; AMAR, M.; SZARFARC, S. C.; DILLMAN, E.; FOSIL, M.; GONGORA BIACHI, R.; GREBE, G.; HERTRAMPF, E.; KREMENCHUZKY, S.; LAYRISSE, M.; MARTINEZ TORRES; C.; MORON, C.; PIZARRO, F.; REYNAFARJE, C.; STEKEL, A.; VILLAVICENCIO, D. \& ZUNIGA, H., 1984. Iron absorption from typical Latin American diets. American Fournal of Clinical Nutrition, 39: 953-962.

AGGETT, P. J.; BARCLAY, S. \& WHITLEY, J. E., 1989. Iron for suckling. Acta Paediatrica Scandinavica, 361 (Suppl.): 96-102.

AUKETT, M. A.; PARKS, Y. A.; SCOTT, P. H. \& WHARTON, B. A., 1986. Treatment with iron increases weight gain and psychomotor development. Archievs of Disease in Childhood, 61: 849-857.

BALLIN, A.; BERAR, M.; RUBINSTEIN, U.; KLETER, Y.; HERSHKOVITZ, A. \& MEYTES, D., 1992. Iron state in female adolescents. American Fournal of Diseases of Children, 146: 803-805.

BATISTA-FILHO, M. \& DINIZ, A. S., 1993. Seminário: Combate às Deficiências de Micronutrientes no Brasil. Relatório Final. Brasília: Instituto Nacional de Alimentação e Nutrição/Organização Panamericana de Saúde. (Mimeo.)

BAYNES, R. D. \& BOTHWELL, T. H., 1990. Iron deficiency. Annual Review of Nutrition, 10: 133-148.

BATES, C. J. \& POWERS, H. J., 1989. Vitamins, iron and physical work. Lancet, 5: 313-314. 
BOTHWELL, T. H.; BAYNES, R. D.; MacFARLANE, B. J. \& MacPHAIL, A. P., 1989. Nutritional iron requirements and food iron absorption. Fournal of Internal Medicine, 226: 357-365.

CALVO, E.; GALINDO, A. C. \& ASPRES, N. B., 1992. Iron status in exclusively breastfed infants. Pediatrics, 90: 375-379.

CARDOSO, M. A.; FERREIRA, M. U.; CAMARGO, L. M. A. \& SZARFARC, S.C., 1992. Anemia em população de área endêmica de malária, Rondônia (Brasil). Revista de Saúde Pública, 26: 161-166.

COOK, J. D.; ALVARADO, J.; GUTNISKY, A.; JAMRA, M.; LABARDINI, J.; LAYRISSE, M.; LINARES, J.; LORIA, A.; MASPES, U.; RESTREPO, A.; REYNAFARJE, C.; SÁNCHEZ-MEDAL, L.; VÉLEZ, H. \& VITERI, F., 1971. Nutritional deficiency and anemia in Latin America: a collaborative study. Blood, 38: 591-603.

COOK, J. D.; CARRIAGA, M.; KAHN, S. G.; SCHALCH, W. \& SKIKNE, B. S., 1990. Gastrie delivery system for iron supplementation. Lancet, 335: 1136-1139.

COOK, J. D. \& REUSSER, M. E., 1983. Iron fortification: an update. American fournal of Clinical Nutrition, 38: 648-659.

DALLMAN, P. R., 1990. Progress in the prevention of iron deficiency in infants. Acta paediatrica Scandinavica, 365 (Suppl.): 28-37.

DE BENAZE, C.; GALAN, P.; WAINER, R. \& HERCBERG, S., 1989. Prévention de l'anémie ferriprive au cours de la grossesse par une supplémentation martiale précoce: un essai contrôlé. Revue d'Epidémiologie et de Santé Publique, 37: 109-118.

DE MAEYER, E. M. \& ADIÉLS-TEGMAN, M., 1985. The prevalence of anaemia in the world. World Health Statistical Quartely, 38: 302-316.

DE MAEYER, E. M.; DALLMAN, P.; GURNEY, J. M.; HALLBERG, L.; SOOD, S. K. \& SRIKANTIA, S. G., 1989. Preventing and Controlling Iron Deficiency Anaemia Through Primary Health Care. Geneva: World Health Organization.
DEVASTHALI, S. D.; GORDEUK, V. R.; BRITTENHAM, G. M.; BRAVO, J. R.; HUGHES, M. A. \& KEATING, L. J., 1991. Bioavailability of carbonyl iron: a randomized, double-blind study. European Fournal of Haematology, 46: 272-278.

FORSYTH, J. S.; OGSTON, S. A.; CLARK, A.; FLOREY, C. V. \& HOWIE, P. W., 1993. Relation between early introduction of solid food to infants and their weight and illnesses during the first two years of life. British Medical fournal, 306: 1572-1576.

GILLESPIE, S.; KEVANY, J. \& MASON, J., 1991. Controlling Iron Deficiency. Geneva: United Nations/Administrative Committee on Coordinations/Subcommittee on Nutrition.

HALLBERG, L. \& ROSSANDER HULTHÉN, L., 1989. Prevalence of iron deficiency in European Countries and attempts to analyze possible causes of differences. In: Nutrition in the Prevention of Disease (J. C. Somogyi \& S. Hejda, eds.) vol. 44, pp. 94-105, Basel: Bibliotheca Nutrition et Dieta.

HASCHKE, F. \& JAVAID, N., 1991. Nutritional anemias. Acta Paediatrica Scandinavica, 374 (Suppl.): 38-44.

HATHIRAT, P.; VALYASEVI, A.; KOTCHABHAKDI, N. J.; ROJROONGWASINKUL, N. \& POLLITT, E., 1992. Effects of an iron supplementation trial on the Fe status of Thai school-children. British Fournal of Nutrition, 68: 245-252.

HERCBERG, S.; GALÁN, P.; CHAULIAC, M.; MASSE-RAIMBAULT, A. M., DEVANLAY, M.; BILEOMA, S; ALIHONOU, E.; ZOHOUN, I.; CHRISTIDES, J. P. \& COURCY, G. P., 1987. Nutritional anaemia in pregnant Beninese women: consequences on the haematological profile of the newborn. British Fournal of Nutrition, 57: 185-193.

HEYWOOD, A.; OPPENHEIMER, S.; HEYWOOD, P. \& JOLLEY, D., 1989. Bechavioral effects of iron supplementation in infants in Madang, Papua New Guinea. American Fournal of Clinical Nutrition, 50: 630-640. 
HUNT, J. R.; MULLEN, L. M.; LYKKEN, G. I.; GALLAGHER, S. K. \& NIELSEN, F. H., 1990. Ascorbic acid: effect on ongoing iron absorption and status in iron-depleted young women. American fournal of Clinical Nutrition, 51: 649-655.

IDJRADINATA, P. \& POLLITT, E., 1993. Reversal of developmental delays in irondeficient anaemic infants treated with iron. Lancet, 341: 01-04.

JAVAID, N.; HASCHKE, F.; PRETSCHNIG, B.; SCHUSTER, E.; HUEMER, C.; SHEBAZ, A; GANESH, P.; STEFAN, I.; HUNEL, R. \& SECRETIN, M. C., 1991. Interactions between infections, malnutrition and iron nutritional status in Pakistani infants. Acta Paediatrica Scandinavica, 374(Suppl.): 141-150.

KASILI, E. G., 1990. Malnutrition and infections as causes of cildhood anemia in tropical Africa. American Fournal of Pediatrics Hematology Oncology, 12: 375-377.

KAUFER, M. \& CASANUEVA, E., 1990. Relation of prepregnancy serum ferritin levels to hemoglobin levels throughout pregnancy. European Fournal of Clinical Nutrition, 44: 709-715.

KLEVAY, L. M., 1992. Clinical signs of iron deficiency. European Fournal of Clinical Nutrition, 46: 607-608.

LERNER, A. \& IANCU, T. C., 1988. Advances in understanding the bioavailability and absorption of iron. In: Frontiers of Gastrointestinal Research - Progress in Diet and Nutrition (C. Horwitz \& P. Rozen, eds.), vol. 14, pp. 117-134, Tel Aviv: Karger.

LIRA, P. I. C.; CARTAGENA, H. A.; ROMANI, S. A. M.; TORRES, M. A. A. \& BATISTAFILHO, M., 1985. Estado nutricional de crianças menores de seis anos, segundo posse da terra, em áreas rurais do Estado de Pernambuco, Nordeste do Brasil. Archivos Latinoamericano de Nutrición, 35: 247-257.

LOZOFF, B.; BRITTENHAM, G. M.; WOLF, A. W.; McCLISH, D. K.; KUHNERT, P. M.; JIMENEZ, E.; JIMENEZ, R.; MORA, L. A.; GOMEZ, I. \& KRAUSKOPH, D., 1987. Iron deficiency anemia and iron therapy. Effects on infant developmental test performance. Pediatrics, 79: 981-95.
LOZOFF, B.; JIMENEZ, E. \& WOLF, A. W., 1991. Long-term developmental outcome of infants with iron deficiency. New England Fournal of Medicine, 325: 687-694.

MASSEY, A. C., 1992. Microcytic anemia. Differential diagnosis and management of iron deficiency anemia. Medical Clinics of North America, 76: 549-566.

MONTEIRO, C. A. \& SZARFARC, S. C., 1987. Estudo das condições de saúde das crianças do município de São Paulo, SP (Brasil) 19841985. V. Anemia. Revista de Saúde Pública, 22: $255-260$.

NOGUEIRA, N. N.; COLLI, C. \& COZZOLINO, S. M. F., 1992. Biscoitos fortificados com sangue bovino. Efeito no estado de nutrição em ferro - estudo preliminar. Cadernos de Saúde Pública, 8: 270286.

OLIVARES, M.; WALTER, T.; HERTRAMPF, E.; PIZARRO, F. \& STEKEL, A., 1989. Prevention of iron deficiency by milk fortification. Acta Paediatrica Scandinavia, 361 (Suppl.): 109-113.

OLIVARES, M.; LLAGUNO, S.; MARIN, V.; HERTRAMPF, E.; MENA, P. \& MILAD, M., 1992. Iron status in low-birth-weight infants, small and appropriate for gestational age. A follow-up study. Acta Paediatrica, 81: 824-828.

OSKI, F. A., 1993. Iron deficiency in infancy and childhood. New England Fournal of Medicine, 15: 190-193.

PIZARRO, F; YIP, R.; DALLMAN, P. R.; OLIVARES, M.; HERTRAMPF, E. \& WALTER, T., 1991. Iron status with different infant feeding regimens: relevance to screening and prevention of iron deficiency. Fournal of pediatrics, 118: 687692.

POLLITT, E.; 1991. Effects of a diet deficient in iron on the growth and development of preschool and school-age children. Food and Nutrition Bulletim, 13: 110-118.

RAMAN, L.; VASUMATHI, N.; RAWAL, A. \& RAJALAKSHMI, K., 1989. Feasibility of parenteral iron therapy as a field approach for management of pregnancy anaemia. Indian Fournal of Medical Research, 90: 258261. 
SALZANO, A. C.; TORRES, M. A. A.; BATISTA-FILHO, M. \& ROMANI, S. A. M., 1985. Anemia em crianças de dois serviços de saúde de Recife, PE (Brasil). Revista de Saúde Pública, 19: 499-507.

SALZANO, A. C.; BATISTA-FILHO, M.; FLORES, H. \& CALADO, C. L. A., 1980. Prevalência de anemia no ciclo gestacional em dois Estados do Nordeste brasileiro, Pernambuco e Paraíba. Revista Brasileira de Pesquisa Médica e Biológica, 13: 211-214.

SARMA, K. V. R.; BRAHMAM, G. N. V.; REDDY, C. G.; RAVINDRANATH, M. \& RAO, N. P., 1992. Iron fortified salt distribution through integrated child development services in Orissa - an assessment. Asia Pacific Fournal of Clinical Nutrition, 1: 239-243.

SCHOLL, T. O.; HEDIGER, M. L.; FISCHER, R. L. \& SHEARER, J. W., 1992. Anemia vs iron deficiency: increased risk of preterm delivery in a prospective study. American Fournal of Clinical Nutrition, 59: 985-988.

SCHULTINK, W.; VAN DER REE, M.; MATULESSI, P. \& GROSS, R., 1993. Low compliance with an iron-supplementation program: a study among pregnant women in Jakarta, Indonesia. American fournal of Clinical Nutrition, 57: 135-139.

SIGULEM, D. M.; TUDISCO, E. S.; GOLDENBERG, P.; ATHAIDE, M. M. M. \& VAISMAN, E., 1978. Anemia ferropriva em crianças do município de São Paulo. Revista de Saúde Pública, 12: 168-178.

SIIMES, M. A. \& ALMENPERÃ, L., 1989. The weanling: iron for all or one. Acta Paediatrica Scandinavia, 361 (Suppl.): 103-108.

SILVA, L. C.; FUENTELSAZ, C. \& AMADOR, M., 1993. Características de la introducción de alimentos al lactante en Cuba, 1993. Boletin de la Oficina Sanitaria Panamericana, 114: 407-414.

SMITH, A. W.; HENDRICKSE, R. G.; HARRISON, C.; HAYES, R. J. \& GREENWOOD, B. M., 1989. Irondeficiency anaemia and its response to oral iron: report of a study in rural Gambian children treated at home by their mothers. Anals of Tropical Pediatrics, 9: 6-16.
SZARFARC, S. C., 1974. Anemia ferropriva em parturientes e recém-nascidos. Revista de Saúde Pública, 8: 369-374.

SZARFARC, S. C., 1985. Anemia nutricional entre gestantes atendidas em Centros de saúde do Estado de São Paulo (Brasil). Revista de Saúde Pública, 19: 450-457.

TROWBRIDGE, F. L.; HARRIS, S. S.; COOK, J.; DUNN, J. T.; FLORENTINO, R. F.; KODYAT, B. A.; MANNAR, M. G. V.; REDDY, V.; TONTISIRIN, K.; UNDERWOOD, B. A. \& YIP, R., 1993. Coordinated strategies for controlling micronutrient malnutrition: a technical workshop. Fournal of Nutrition, 123: 775-787.

VANNUCCHI, H.; FREITAS, M. L. S. \& SZARFARC, S. C., 1992. A prevalência de anemias nutricionais no Brasil. Cadernos de Nutrição, 4: 07-26.

WALTER, T., 1993. Impact of iron deficiency on cognition in infancy and childhood. European Fournal of Clinical Nutrition, 47: 307-316.

WALTER, T.; DE ANDRACA, I.; CHADUD, P. \& PERALES, C. G., 1989. Iron deficiency anemia: adverse effects on infant psychomotor developmente. Pediatrics, 84: 7-17.

WALTER, T.; DALLMAN, P. R.; PIZARRO, F.; VELOZO, L.; PENÃ, G.; BARTHOLMEY, S. J.; HERTRAMPF, E.; OLIVARES, M.; LETELIER, A. \& ARREDONDO, M., 1993a. Effectiveness of iron-fortified infant cereal in prevention of iron deficiency anemia. Pediatrics, 91: 976982.

WALTER, T.; HERTRAMPF, E.; PIZARRO, F.; OLIVARES, M.; LLAGUNO, S.; LETELIER, A.; VEJA, V. \& STEKEL, A., 1993b. Effect of bovine-hemoglobinfortified cookies on iron status of schoolchildren: a nationwide program in Chile. American fournal of Clinical Nutrition, 57: 190-194.

YIP, R.; REEVES, J. D.; LÖINNERDAL, B.; KEEN, C. L. \& DALLMAN, P. R., 1985. Does iron supplementation compromise zinc nutrition in healthy infants? American Fournal of Clinical Nutrition, 42: 683-687. 